\title{
Plasma miR-208b and miR-499: Potential Biomarkers for Severity of Coronary Artery Disease
}

\author{
Wei Wang, ${ }^{1}$ Tai $\mathrm{Li}^{2}{ }^{2}$ Lei Gao, ${ }^{3}$ Yang $\mathrm{Li}^{3}{ }^{3}$ Ying Sun, ${ }^{1}$ and Heng-Chen Yao ${ }^{1}$ \\ ${ }^{1}$ Department of Cardiology, Liaocheng People's Hospital Affiliated to Shandong University and Clinical School of Shandong First \\ Medical University, Liaocheng 252000, China \\ ${ }^{2}$ Department of Nursing, Liaocheng Vocational and Technical College, Liaocheng 252000, China \\ ${ }^{3}$ Zhong Yuan Academy of Biological Medicine, Liaocheng People's Hospital, Shandong University, Liaocheng, China
}

Correspondence should be addressed to Heng-Chen Yao; yaohc66@hotmail.com

Received 26 July 2019; Revised 7 November 2019; Accepted 11 November 2019; Published 22 November 2019

Academic Editor: Monica Cantile

Copyright (c) 2019 Wei Wang et al. This is an open access article distributed under the Creative Commons Attribution License, which permits unrestricted use, distribution, and reproduction in any medium, provided the original work is properly cited.

\begin{abstract}
Aims. MicroRNAs (miRNAs) are associated with the pathogenesis of coronary artery disease (CAD). The objective of this study is to explore plasma levels of miR-208b and miR-499 in CAD and analyze its association with the severity of CAD. Materials and Methods. 195 consecutive CAD patients who underwent coronary angiography were enrolled in this study. Severity of coronary lesions was evaluated by the synergy between percutaneous coronary intervention with taxus and cardiac surgery score (SYNTAX) score (SS). Plasma levels of miR-208b and miR-499 were assessed by quantitative real-time polymerase chain reaction (qRT-PCR). The relationship between miR-208b and miR-499 and SS was analyzed. Results. The qRT-PCR results showed that plasma levels of miR-208b and miR-499 in SS $>32$ (high SS) group was higher than those in low (SS $\leq 22$ ) and intermediate $(22<\mathrm{SS} \leq 32)$ groups. Meanwhile, plasma miR-208b and miR-499 levels were significantly positive correlated with the SS (Spearman's $r=0.535$ and $r=0.407$, respectively; both $p<0.001$ ). Multivariate logistic analysis results showed that miR-208b (odds ratio [OR]: 2.069; 95\% confidence interval [CI]: 1.351-3.167; $p=0.001$ ) and miR-499 (OR: 1.652; 95\% CI: 1.222-2.233; $p=0.001$ ) were independent predictors of high SS. In receiver operating characteristic curve, the area under the curve of miR-208b and miR-499 in prediction of high SS was 0.775 and 0.713, respectively. Conclusions. Higher plasma levels of miR-208b and miR-499 were positively associated with the severity of CAD, and plasma miR-208b and miR-499 can act as potential biomarkers for estimating the severity of CAD.
\end{abstract}

\section{Introduction}

Coronary artery disease (CAD) and its clinical complications is still a major cause of death in the world $[1,2]$. The severity of CAD is closely associated with mortality, and the synergy between percutaneous coronary intervention with taxus and cardiac surgery score (SYNTAX) score (SS) was used to determine CAD severity by scoring system. SS has been proved to predict clinical outcomes not only in acute coronary syndrome (ACS) but also in stable CAD [3-5]. Identifying relevant factors associated with high SS and close monitoring during and after hospitalization can improve the prognosis of CAD patients. Various plasma biomarkers have been proved to associate with CAD, but very few of them can provide valid information about the severity of
CAD [6-8]. Therefore, more new biomarkers are needed to assess the severity of CAD.

Several lines of evidence showed that microRNAs (miRNAs) regulate signaling pathways involved in cell proliferation and differentiation, which plays an important role in the pathogenesis of atherosclerosis [9-11]. Plasma miRNAs could be considered as promising biomarkers for the diagnosis and prognosis of these diseases, especially for CAD $[12,13]$. miR-208b and miR-499 are involved in cardiogenesis, and they are the main regulators of left ventricular remodeling and cardiac hypertrophy and involved in mediating the differentiation of cardioblasts to cardiomyocytes [14-16]. Additionally, studies have revealed that miR-208b is highly expressed during myocardial infarction (MI) [17]. Zhang et al. found that the expression of downregulated 
miR-499 increased PDCD4 expression and protected endothelial cells (ECs) from inflammatory damage during CAD by the nuclear factor-kappa $\mathrm{B}(\mathrm{NF}-\kappa \mathrm{B}) /$ tumor necrosis factor-alpha (TNF- $\alpha$ ) signaling pathway [18].

Although the role of miR-208b and miR-499 in the blood vessels has been reported, the association between levels of circulating miR-208b and miR-499 and severity of CAD has not been studied yet. In the present study, we aimed to evaluate the relationship of plasma miR-208b and miR-499 levels with CAD severity.

\section{Methods}

2.1. Study Population. Between April and November 2016, 195 consecutive patients with CAD who underwent coronary angiography (CAG) in Liaocheng People's Hospital were enrolled in this study. Diagnostic criteria were referred to ACCF/AHA Guideline for the Management of ST-elevation Myocardial Infarction or Non-ST-Elevation Acute Coronary Syndromes $[19,20]$. The inclusion criteria were patients with CAD aged from 35 to 85 years, and the chest pain lasting $<24$ hours. The exclusion criteria were patients with known inflammatory disease, valvular heart disease and received anticoagulant and patients who had significant hepatic dysfunction or renal failure, cancer, or malignancy and had diseases of hematological and immune system. After these exclusions, 195 patients were enrolled. According to hospital records, baseline characteristics and past medical history including hypertension, diabetes mellitus, and smoking status were collected. The SS was determined by two experienced cardiologists who were blinded to the laboratory and clinical data of patients. Patients were divided into three groups according to the SS levels as following: high SS $>32$, $22<$ intermediate $\mathrm{SS} \leq 32$, and low $\mathrm{SS} \leq 22$. The study protocol was approved by the Medical Ethics Committee of Liaocheng People's Hospital. All procedures were in accordance with principles of Helsinki Declaration, and all patients provided informed consent.

2.2. Sample Collection and Storage. After admission, blood collection was performed from study subjects prior to the CAG procedures. Blood samples were collected using ethylenediaminetetraacetic acid tubes. After centrifugation, samples were transferred to RNase/DNase-free tubes and stored at $-80^{\circ} \mathrm{C}$.

2.3. Plasma MicroRNA Isolation and Validation. Total RNA was extracted using TRIzol reagent (Invitrogen, USA), and PrimeScript ${ }^{\mathrm{TM}}$ RT Master Mix (Takara, Japan) was used for the reverse transcription reaction according to the manufacturer's instructions. Quantitative real-time polymerase chain reaction was conducted using $\mathrm{SYBR}^{\circledR}$ PrimeScript $^{\mathrm{TM}}$ miRNA RT-PCR Kit (Takara, Japan). Cycle threshold (Ct) values were normalized to cel-miR-39 using the formula $2^{-(\mathrm{Ct}[\mathrm{miR}]-\mathrm{Ct}[\mathrm{cel}-\mathrm{miR}-39])}$ and the relative expression levels of miRs were analyzed by the $2^{-\Delta \Delta \mathrm{Ct}}$ method.

2.4. Statistical Analysis. Statistical analysis was performed using SPSS 23.0 version software (IBM Corp.). Shapiro-Wilk test was used to verify whether continuous data was normally distribution. Normally distributed data was expressed as mean \pm standard deviation, and nonnormal distribution was presented as median (quartile deviation). Categorical variables were presented as counts (percentage). One-way analysis of variance (ANOVA) or the Kruskal-Wallis $H$ test was used for comparisons among three groups. Chi-squared test was used to compare the categorical data. To analyze the differences of miR-208b and miR-499 expression intergroup, Steel-Dwass multiple comparisons tests were used for nonparametric variables post hoc analysis. Spearman's correlation test was used to analyze the correlation of SS and miR-208b and miR-499. Independent factors for predicting high SS were calculated by univariate analysis; variables with $p$ value of $<0.05$ in univariate analysis were included in multivariate logistic regression models. Meanwhile, to further explore the applicability of using circulating miR-208b and miR-499 in predicting high SS, we performed receiver operating characteristic (ROC) curve analyses. All analyses were two-sided; the $p$ value of $<0.05$ was determined as statistical significant. Steel-Dwass test was performed with free software (MEPHAS) available at http://www.gen-info.osaka-u. ac.jp/testdocs/tomocom/.

\section{Results}

3.1. Patient Characteristics. The study including 195 CAD patients who underwent CAG and the basic characteristics of patients are shown in Table 1. There were no significant differences in the proportion of age, gender, smoking status, and diabetes mellitus in the 3 groups. Laboratory parameters showed the levels of creatinine ( $\mathrm{Cr}$ ), creatine kinase isoenzymes (CK-MB), and high sensitivity C-reactive protein (hs-CRP) were higher in higher SS group than in lower SS group, as well as the mortality rate during hospitalization.

3.2. Plasma Levels of miR-208b and miR-499 Were Positively Correlated with the Severity of CAD. Patients with higher SS had significantly higher miR-208b and miR-499 levels compared with those with lower SS $(p<0.05)$ (Figure 1 and Table 2). Meanwhile, plasma miR-208b and miR-499 levels were positively associated with SS (Spearman's $r=0.535$ and $r=0.407$, respectively; both $p<0.001$ ) (Figure 2). These results that indicated the increased expression of plasma miR-208b and miR-499 is significantly associated with the severity of CAD.

3.3. $m i R-208 b$ and miR-499 Were Independent Predictors of the Severity of $C A D$. Multivariate logistic regression analysis was used to reveal the independent predictors of high SS using variables that showed statistically significant association in the univariate analysis. The result showed that miR208b (odds ratio [OR]: 2.069; 95\% confidence interval [CI]: 1.351-3.167; $p=0.001$ ) and miR-499 (OR: 1.652; 95\% CI: $1.222-2.233 ; p=0.001)$ were independent predictors of high SS (Table 3 ).

3.4. Performance of miR-208b and miR-499 in the Prediction of Severity of CAD. To evaluate the performance of miR-208b and miR-499 in the prediction of high SS, ROC curves were 
TABLE 1: Characteristics of patients according to SYNTAX score level.

\begin{tabular}{|c|c|c|c|c|}
\hline & $\mathrm{SS} \leq 22(n=43)$ & $22<\mathrm{SS} \leq 32(n=72)$ & SS $>32(n=80)$ & $p$ value \\
\hline Age (year) & $60.19 \pm 11.21$ & $63.29 \pm 10.25$ & $64.58 \pm 9.95$ & 0.082 \\
\hline Male gender, $n(\%)$ & $28(65.12)$ & $41(56.94)$ & $49(61.25)$ & 0.676 \\
\hline Smoking, $n(\%)$ & $12(27.91)$ & $23(31.94)$ & $37(46.25)$ & 0.072 \\
\hline Hypertension, $n(\%)$ & $19(44.19)$ & $51(70.83)$ & $51(63.75)$ & 0.016 \\
\hline $\mathrm{DM}, n(\%)$ & $5(11.63)$ & $15(20.83)$ & $23(28.75)$ & 0.088 \\
\hline Heart rate (bpm) & $72.00(12.00)$ & $74.86 \pm 11.46$ & $78.00(19.75)$ & 0.025 \\
\hline Hemoglobin $(\mathrm{g} / \mathrm{L})$ & $136.28 \pm 14.91$ & $137.56 \pm 16.08$ & $133.71 \pm 14.10$ & 0.281 \\
\hline Leukocyte $\left(\times 10^{9} / \mathrm{dL}\right)$ & $6.09 \pm 1.58$ & $6.17 \pm 1.45$ & $7.24(2.56)$ & $<0.001$ \\
\hline Creatinine $(\mu \mathrm{mol} / \mathrm{L})$ & $65.79 \pm 12.84$ & $70.15 \pm 17.68$ & $67.79 \pm 13.97$ & 0.036 \\
\hline $\mathrm{HDL}(\mathrm{mmol} / \mathrm{L})$ & $1.28(0.36)$ & $1.25(0.35)$ & $1.23 \pm 0.25$ & 0.031 \\
\hline $\mathrm{LDL}(\mathrm{mmol} / \mathrm{L})$ & $2.66 \pm 0.48$ & $2.69 \pm 0.74$ & $2.59 \pm 0.71$ & 0.621 \\
\hline TC (mmol/L) & $4.80 \pm 1.05$ & $4.64(1.83)$ & $4.52(1.18)$ & 0.742 \\
\hline CK-MB (IU/L) & $11.51 \pm 7.24$ & $10.00(4.75)$ & $17.50(16.50)$ & $<0.001$ \\
\hline Hs-CRP (mg/L) & $1.04(1.98)$ & $0.88(3.04)$ & $3.84(13.94)$ & $<0.001$ \\
\hline SS & $12.00(15.00)$ & $27.00(5.00)$ & $43.5(12.00)$ & $<0.001$ \\
\hline In-hospital mortality, $n(\%)$ & $2(4.65)$ & $2(2.78)$ & $11(13.75)$ & 0.028 \\
\hline
\end{tabular}

Abbreviation: SYNTAX: synergy between percutaneous coronary intervention with taxus and cardiac surgery; SS: SYNTAX score; DM: diabetes mellitus; HDL: high-density lipoprotein; LDL: low-density lipoprotein; TC: total cholesterol; CK-MB: creatine kinase isoenzyme; Hs-CRP: high-sensitivity C-reactive protein.

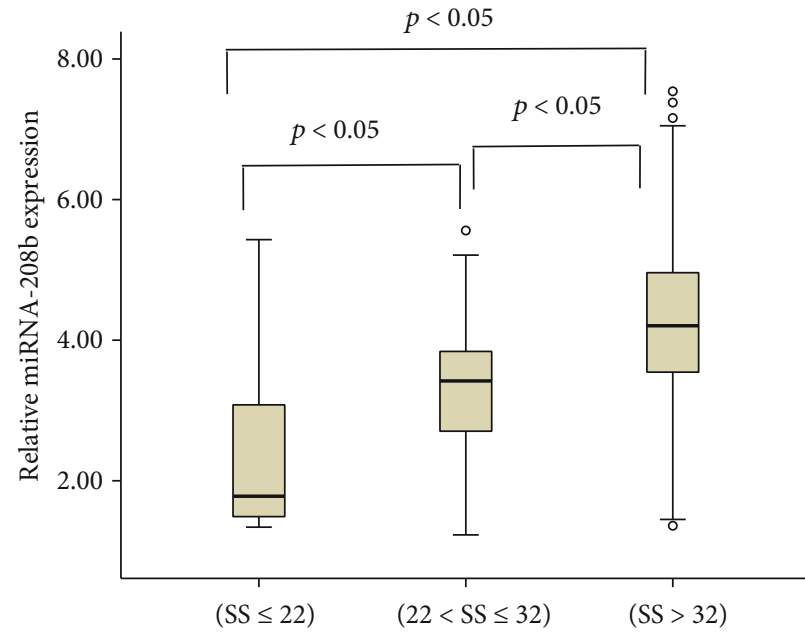

(a)

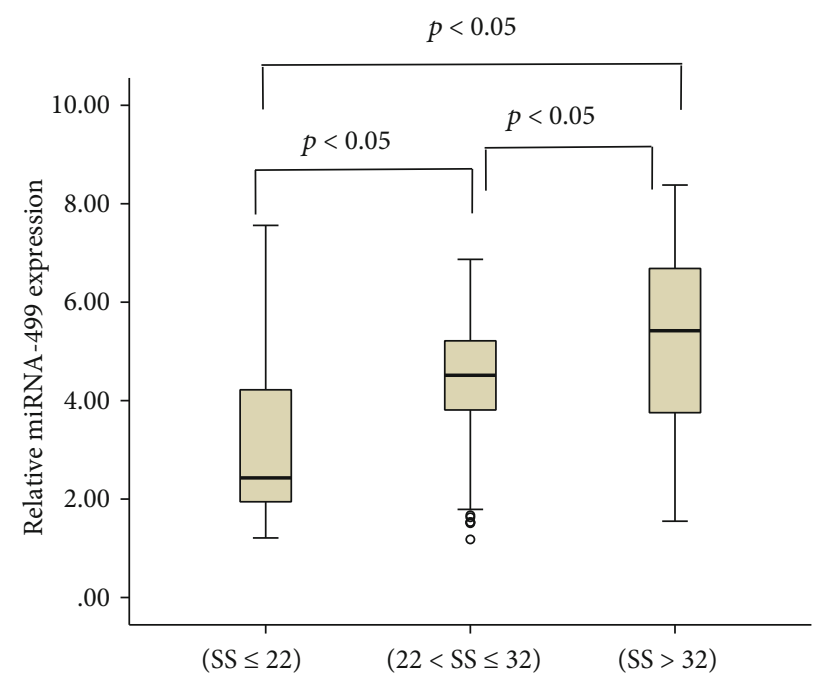

(b)

FIGURE 1: Relative expression of miR-208b (a) and miR-499 (b) in three groups. Abbreviation: SS: synergy between percutaneous coronary intervention with taxus and cardiac surgery (SYNTAX) score.

TABLE 2: Expression levels of circulating miRNA-208b and miRNA499 in studied patients.

\begin{tabular}{lccc}
\hline Variables & $\begin{array}{c}\mathrm{SS} \leq 22 \\
n=43\end{array}$ & $\begin{array}{c}22<\mathrm{SS} \leq 32 \\
n=72\end{array}$ & $\begin{array}{c}\mathrm{SS}>32 \\
n=80\end{array}$ \\
\hline miRNA-208b & $1.78(1.62)$ & $3.32 \pm 0.84^{*}$ & $4 . .25 \pm 1.33^{* * *}$ \\
miRNA-499 & $2.43(2.44)$ & $4.52(1.48)^{*}$ & $5.42(2.99)^{* * *}$ \\
\hline
\end{tabular}

${ }^{*} p<0.05$ when compared to $22<\mathrm{SS} \leq 32$ or SS $>32$ with $\mathrm{SS} \leq 22 .{ }^{* *} p<0.05$ when compared to $22<\mathrm{SS} \leq 32$ with $\mathrm{SS}>32$. Abbreviation: SS: synergy between percutaneous coronary intervention with taxus and cardiac surgery score. performed (Figure 3). The area under the curve (AUC) values were $0.775(p<0.001)$ for miR-208b with $59 \%$ sensitivity and $88 \%$ specificity and the optimal cutoff value was 3.95 ; $0.713(p<0.001)$ for miR-499 with 54\% sensitivity and $91 \%$ specificity and the optimal cutoff value was 5.19 in predicting high SS.

\section{Discussion}

According to our findings, we found that increased levels of miR-208b and miR-499 were significantly associated with 


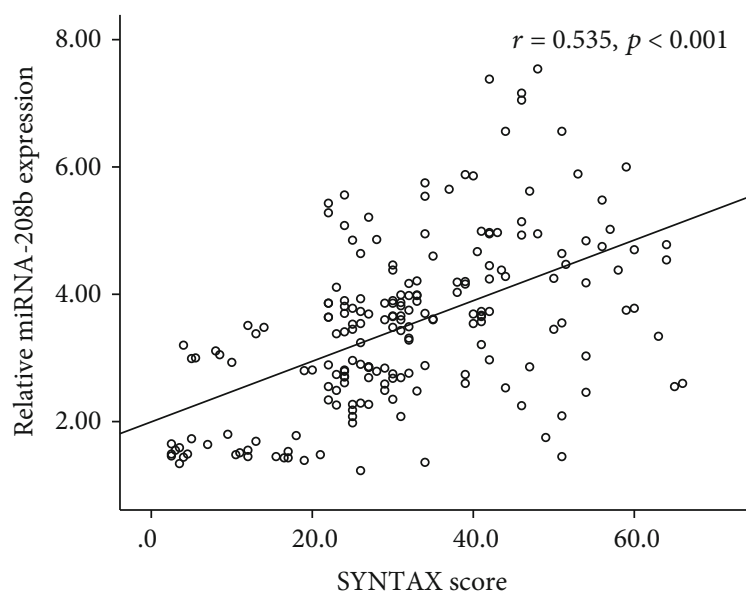

(a)

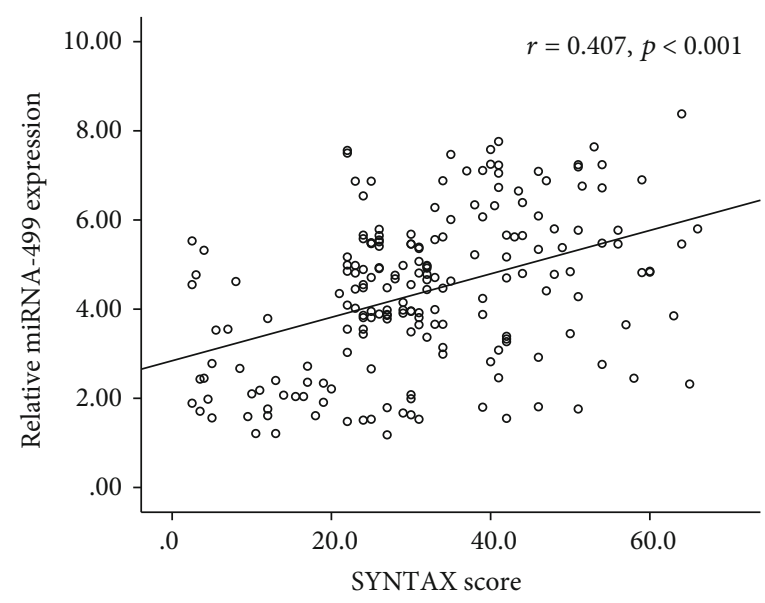

(b)

FIGURE 2: Correlation graph of miR-208b (a) and miR-499 (b) with SYNTAX score. Abbreviation: SYNTAX: synergy between percutaneous coronary intervention with taxus and cardiac surgery.

TABLE 3: Logistic regression analysis for determining the independent predictors of high SS.

\begin{tabular}{|c|c|c|c|c|c|c|}
\hline Variables & OR & $95 \% \mathrm{CI}$ & $p$ value & Adjusted OR & $95 \% \mathrm{CI}$ & $p$ value \\
\hline Male gender & 0.949 & $0.529-1.702$ & 0.861 & & & \\
\hline Age & 1.023 & $0.995-1.052$ & 0.109 & & & \\
\hline Hypertension & 1.113 & $0.627-2.039$ & 0.684 & & & \\
\hline Diabetes mellitus & 1.917 & $0.968-3.796$ & 0.062 & & & \\
\hline Current smoking & 1.967 & $1.088-3.557$ & 0.025 & 2.019 & $0.805-5.064$ & 0.134 \\
\hline Heart rate & 1.036 & $1.013-1.060$ & 0.002 & 1.031 & 0.994-1.069 & 0.099 \\
\hline Hemoglobin & 0.985 & $0.968-1.004$ & 0.126 & & & \\
\hline Leukocyte & 1.472 & $1.230-1.761$ & $<0.001$ & 1.355 & $1.051-1.747$ & 0.019 \\
\hline Hs-CRP & 1.045 & $1.017-1.074$ & 0.001 & 1.043 & $1.003-1.085$ & 0.035 \\
\hline Creatinine & 0.997 & $0.978-1.016$ & 0.743 & & & \\
\hline CK-MB & 1.125 & $1.067-1.185$ & $<0.001$ & 1.129 & $1.054-1.209$ & 0.001 \\
\hline LDL-cholesterol & 0.814 & $0.530-1.251$ & 0.349 & & & \\
\hline HDL-cholesterol & 0.215 & $0.069-0.664$ & 0.008 & 0.336 & $0.060-1.884$ & 0.215 \\
\hline Total cholesterol & 0.987 & $0.774-1.259$ & 0.919 & & & \\
\hline miRNA-208b & 2.553 & $1.863-3.499$ & $<0.001$ & 2.069 & $1.351-3.167$ & 0.001 \\
\hline miRNA-499 & 1.644 & $1.347-2.008$ & $<0.001$ & 1.652 & $1.222-2.233$ & 0.001 \\
\hline
\end{tabular}

Abbreviation: SS: synergy between percutaneous coronary intervention with taxus and cardiac surgery score; OR: odds ratio; CI: confidence interval; Hs-CRP: high-sensitivity C-reactive protein; CK-MB: creatine kinase isoenzyme; LDL: low-density lipoprotein; HDL: high-density lipoprotein.

CAD severity. All we know, this is the first study to report that miR-208b and miR-499 levels are independent predictors of high SS.

SS is usually used for calculating the severity and complexity of CAD and as a guideline for interventional cardiologist to choose more appropriate treatment between percutaneous coronary intervention (PCI) and coronary artery bypass graft surgery [21]. There is increasing evidence that has proven that this scoring system could predict major adverse cardiovascular events in patients with ACS or undergoing PCI $[22,23]$. As in line with previous studies, in the present study, patients with higher SS have higher inhospital mortality rate than patients with lower SS [7]. One of fundamental characteristics of atherogenesis is chronic inflammation and C-reactive protein (CRP) can reflect the inflammatory status. Previous study has demonstrated a close relationship between SS and inflammation [24]. Karadeniz and colleagues showed that hs-CRP level is associated with intermediate and high SS in patients with ACS [25]. In accordance with these findings, we observed that hs-CRP levels were significantly elevated in higher SS values and hs-CRP was an independent predictor of high SS. The identification of factors correlated with high SS may improve the prognosis of CAD patients. In our study, elevated CK-MB and leukocyte were also independent predictors of high SS. The relationship between $\mathrm{CK}-\mathrm{MB}$, leukocyte, and CAD 


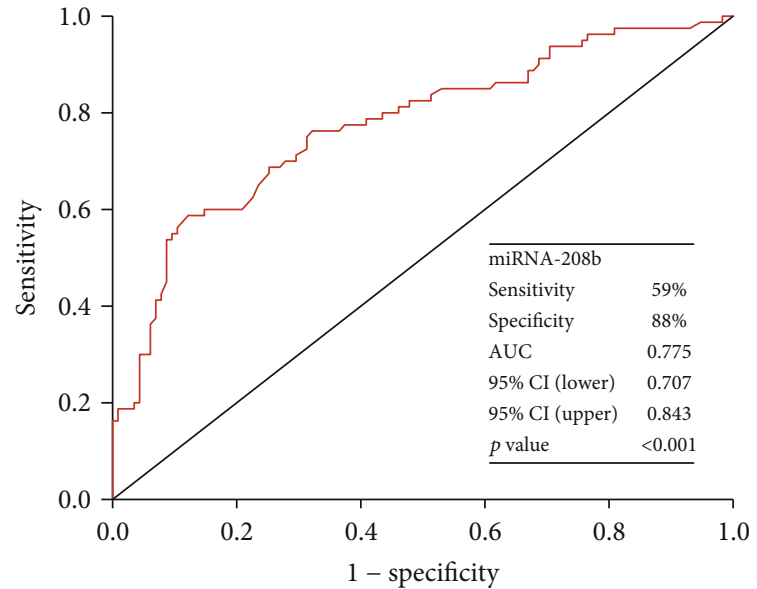

(a)

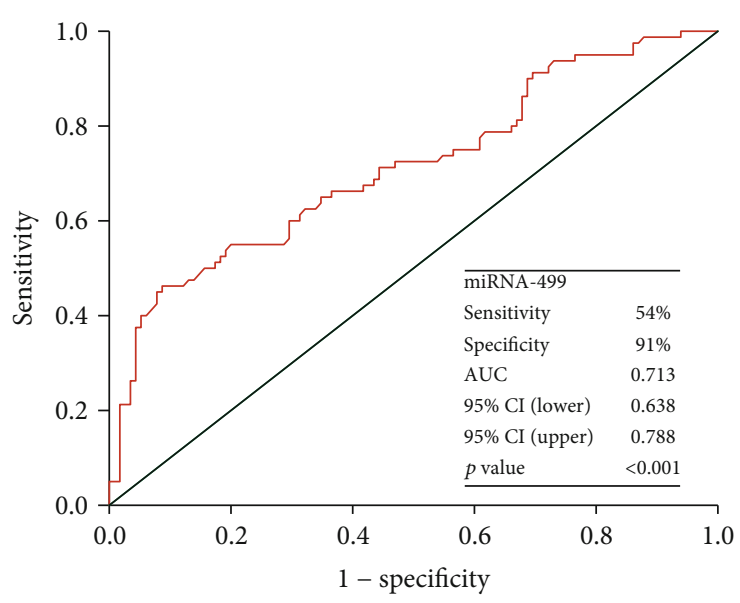

(b)

FIGURE 3: The receiver operating characteristic (ROC) curves of miR-208b (a) and miR-499 (b) in the prediction of high SYNTAX score. Abbreviation: SYNTAX: synergy between percutaneous coronary intervention with taxus and cardiac surgery; AUC: area under the curve; CI: confidence interval.

severity has been established in previous studies $[26,27]$. However, up to date, no study has investigated the association between miR-208b and miR-499 and severity of CAD. Our study indicated that miR-208b and miR-499 might be novel biomarkers for the severity of CAD.

Some pathological changes, including dysfunction of VSMCs and ECs, contribute to the formation of atherosclerosis [28]. VSMCs play a pivotal role in early progression of atherogenesis, which are the first cells present in atherosclerotic plaques. They secrete extracellular matrix that traps lipid from the bloodstream and can take up this lipid to form foam-like cells [29]. Arterial wall EC dysfunction could provoke monocyte adhesion initially, then followed by macrophage intruding the subendothelial area to form foam cells [28]. Circulating miR-208 is a cardiac-rich miRNA, presenting in two isoforms: miR-208a and miR-208b [30]. Zhang et al. [31] reported that miR-208 promotes VSMC proliferation via downregulation of its potential target-p21; in contrast, inhibition of miR-208 can reduce the effect on VSMC proliferation. Paradoxically, Zhou and coworkers found that miR-208b is highly expressed during MI and exerts its myocardioprotective effect against hypoxia-induced apoptosis, which can reduce EC apoptosis and attenuate atherosclerosis development [17, 32]. Further studies should be carried out to promote the understanding of the miR-208b functions in atherosclerosis. Recent study has shown that the expression of downregulated miR-499 increased PDCD4 expression and protected ECs from inflammatory damage during CAD through the NF- $\kappa \mathrm{B} / \mathrm{TNF}-\alpha$ signaling pathway [18]. Moreover, miR-499 overexpression suppressed its target gene-calcineurin A, which could induce EC inflammation and dysfunction $[33,34]$. In our study, we observed that increased levels of miR-208b and miR-499 were significantly associated with higher SS, indicating that the two miRNAs could be potential targets for the treatment of CAD. Certainly, further studies that directly indicate the association of miR-208b and miR-499 and severity of CAD are warranted.
There may be several limitations in our study. First, it is a single-center study which involved a small sample size, and therefore; the predictive value of this study should be interpreted with caution. Second, in our study, we did not elucidate the mechanisms behind the association between circulating levels of miR-208b and miR-499 and severity of CAD. Therefore, larger clinical studies and some biological researches are required.

Overall, circulating miR-208b and miR-499 levels are significantly higher in higher SS patients compared to those in lower SS patients. miR-208b and miR-499 were found to be independent predictors of high SS. Our study may provide further evidence in the prediction of CAD severity for clinical implications.

\section{Data Availability}

The data used to support the findings of this study are available from the corresponding author upon request.

\section{Conflicts of Interest}

The authors declare no conflict of interest.

\section{Authors' Contributions}

Wei Wang and Tai Li contributed equally.

\section{Acknowledgments}

This work was supported by the Traditional Chinese Medicine Science and Technology Development Plan Project of Shandong Province (No. 2019-0891).

\section{References}

[1] R. W. Yeh, S. Sidney, M. Chandra, M. Sorel, J. V. Selby, and A. S. Go, "Population trends in the incidence and outcomes 
of acute myocardial infarction," The New England Journal of Medicine, vol. 362, no. 23, pp. 2155-2165, 2010.

[2] D. Mozaffarian, E. J. Benjamin, A. S. Go et al., "Heart disease and stroke statistics-2015 update: a report from the American Heart Association," Circulation, vol. 131, no. 4, pp. e29-322, 2015.

[3] W. J. van Gaal, F. A. Ponnuthurai, J. Selvanayagam et al., "The syntax score predicts peri-procedural myocardial necrosis during percutaneous coronary intervention," International Journal of Cardiology, vol. 135, no. 1, pp. 60-65, 2009.

[4] T. Palmerini, P. Genereux, A. Caixeta et al., "Prognostic value of the SYNTAX score in patients with acute coronary syndromes undergoing percutaneous coronary intervention: analysis from the ACUITY (Acute Catheterization and Urgent Intervention Triage StrategY) trial," Journal of the American College of Cardiology, vol. 57, no. 24, pp. 2389-2397, 2011.

[5] M. Magro, S. Nauta, C. Simsek et al., "Value of the SYNTAX score in patients treated by primary percutaneous coronary intervention for acute ST-elevation myocardial infarction: the MI SYNTAX score study," American Heart Journal, vol. 161, no. 4, pp. 771-781, 2011.

[6] R. J. Widmer, L. O. Lerman, and A. Lerman, "MicroRNAs: small molecule, big potential for coronary artery disease," European Heart Journal, vol. 37, no. 22, pp. 1750-1752, 2016.

[7] M. Cagdas, I. Rencuzogullari, S. Karakoyun et al., "Assessment of relationship between $\mathrm{C}$-reactive protein to albumin ratio and coronary artery disease severity in patients with acute coronary syndrome," Angiology, vol. 70, no. 4, pp. 361-368, 2019.

[8] A. Berezin, A. Zulli, S. Kerrigan, D. Petrovic, and P. Kruzliak, "Predictive role of circulating endothelial-derived microparticles in cardiovascular diseases," Clinical Biochemistry, vol. 48, no. 9, pp. 562-568, 2015.

[9] D. P. Bartel, "MicroRNAs: genomics, biogenesis, mechanism, and function," Cell, vol. 116, no. 2, pp. 281-297, 2004.

[10] A. Schober, T. Thum, and A. Zernecke, "MicroRNAs in vascular biology-metabolism and atherosclerosis," Thrombosis and Haemostasis, vol. 107, no. 04, pp. 603-604, 2012.

[11] B. Schroen and S. Heymans, "Small but smart-microRNAs in the centre of inflammatory processes during cardiovascular diseases, the metabolic syndrome, and ageing," Cardiovascular Research, vol. 93, no. 4, pp. 605-613, 2012.

[12] C. Li, F. Pei, X. Zhu, D. D. Duan, and C. Zeng, "Circulating microRNAs as novel and sensitive biomarkers of acute myocardial infarction," Clinical Biochemistry, vol. 45, no. 10-11, pp. 727-732, 2012.

[13] M. Li and J. Zhang, "Circulating microRNAs: potential and emerging biomarkers for diagnosis of cardiovascular and cerebrovascular diseases," BioMed Research International, vol. 2015, Article ID 730535, 9 pages, 2015.

[14] D. A. Chistiakov, A. N. Orekhov, and Y. V. Bobryshev, "Cardiac-specific miRNA in cardiogenesis, heart function, and cardiac pathology (with focus on myocardial infarction)," Journal of Molecular and Cellular Cardiology, vol. 94, pp. 107-121, 2016.

[15] T. Fernandes, V. G. Barauna, C. E. Negrao, M. I. Phillips, and E. M. Oliveira, "Aerobic exercise training promotes physiological cardiac remodeling involving a set of microRNAs," American Journal of Physiology Heart and Circulatory Physiology, vol. 309, no. 4, pp. H543-H552, 2015.

[16] P. Lv, M. Zhou, J. He et al., "Circulating miR-208b and miR-34a are associated with left ventricular remodeling after acute myocardial infarction," International Journal of Molecular Sciences, vol. 15, no. 4, pp. 5774-5788, 2014.

[17] Y. L. Zhou, Q. Sun, L. Zhang, and R. Li, "miR-208b targets Bax to protect H9c2 cells against hypoxia-induced apoptosis," Biomedicine \& Pharmacotherapy, vol. 106, pp. 1751-1759, 2018.

[18] Y. H. Zhang, K. He, and G. Shi, "Effects of microRNA-499 on the inflammatory damage of endothelial cells during coronary artery disease via the targeting of PDCD4 through the NF-K $\beta /$ TNF- $\alpha$ signaling pathway," Cellular Physiology and Biochemistry, vol. 44, no. 1, pp. 110-124, 2017.

[19] E. A. Amsterdam, N. K. Wenger, R. G. Brindis et al., "2014 AHA/ACC guideline for the management of patients with non-ST-elevation acute coronary syndromes: a report of the American College of Cardiology/American Heart Association task force on practice guidelines," Journal of the American College of Cardiology, vol. 64, no. 24, pp. e139-e228, 2014.

[20] P. T. O'Gara, F. G. Kushner, D. D. Ascheim et al., "2013 ACCF/AHA guideline for the management of ST-elevation myocardial infarction: a report of the American College of Cardiology Foundation/American Heart Association task force on practice guidelines," Journal of the American College of Cardiology, vol. 61, no. 4, pp. e78-e140, 2013.

[21] S. J. Head, P. M. Davierwala, P. W. Serruys et al., "Coronary artery bypass grafting vs. percutaneous coronary intervention for patients with three-vessel disease: final five-year followup of the SYNTAX trial," European Heart Journal, vol. 35, no. 40, pp. 2821-2830, 2014.

[22] C. G. Braga, A. B. Cid-Alvarez, A. R. Dieguez et al., "Prognostic impact of residual SYNTAX score in patients with ST-elevation myocardial infarction and multivessel disease: Analysis of an 8-year all- comers registry," International Journal of Cardiology, vol. 243, pp. 21-26, 2017.

[23] M. Minamisawa, T. Miura, H. Motoki et al., "Prediction of 1-year clinical outcomes using the SYNTAX score in patients with prior heart failure undergoing percutaneous coronary intervention: sub-analysis of the SHINANO registry," Heart and Vessels, vol. 32, no. 4, pp. 399-407, 2017.

[24] A. G. Ertem, T. H. Efe, C. Yayla et al., "The association between serum procalcitonin levels and severity of coronary artery disease assessed by SYNTAX score in patients with acute coronary syndrome," Angiology, vol. 68, no. 1, pp. 40-45, 2017.

[25] M. Karadeniz, M. Duran, A. Akyel et al., "High sensitive CRP level is associated with intermediate and high SYNTAX score in patients with acute coronary syndrome," International Heart Journal, vol. 56, no. 4, pp. 377-380, 2015.

[26] V. Farooq, P. W. Serruys, P. Vranckx et al., "Incidence, correlates, and significance of abnormal cardiac enzyme rises in patients treated with surgical or percutaneous based revascularisation: a substudy from the synergy between percutaneous coronary interventions with Taxus and cardiac surgery (SYNTAX) trial," International Journal of Cardiology, vol. 168, no. 6, pp. 5287-5292, 2013.

[27] L. F. Hong, X. L. Li, S. H. Luo et al., "Relation of leukocytes and its subsets counts with the severity of stable coronary artery disease in patients with diabetic mellitus," PLoS One, vol. 9, no. 3, article e90663, 2014.

[28] H. Lu and A. Daugherty, "Atherosclerosis," Arteriosclerosis, Thrombosis, and Vascular Biology, vol. 35, no. 3, pp. 485491, 2015.

[29] A. C. Doran, N. Meller, and C. A. McNamara, "Role of smooth muscle cells in the initiation and early progression of 
atherosclerosis," Arteriosclerosis, Thrombosis, and Vascular Biology, vol. 28, no. 5, pp. 812-819, 2008.

[30] Y. Huang and J. Li, "MicroRNA208 family in cardiovascular diseases: therapeutic implication and potential biomarker," Journal of Physiology and Biochemistry, vol. 71, no. 3, pp. 479-486, 2015.

[31] Y. Zhang, Y. Wang, X. Wang et al., "Insulin promotes vascular smooth muscle cell proliferation via microRNA-208-mediated downregulation of p21," Journal of Hypertension, vol. 29, no. 8, pp. 1560-1568, 2011.

[32] M. Qin, Y. Luo, X. B. Meng et al., "Myricitrin attenuates endothelial cell apoptosis to prevent atherosclerosis: an insight into PI3K/Akt activation and STAT3 signaling pathways," Vascular Pharmacology, vol. 70, pp. 23-34, 2015.

[33] S. K. Chua, B. W. Wang, L. M. Lien, H. M. Lo, C. Z. Chiu, and K. G. Shyu, "Mechanical stretch inhibits microRNA499 via p53 to regulate calcineurin-a expression in rat cardiomyocytes," PLoS One, vol. 11, no. 2, article e0148683, 2016.

[34] R. Rodrigues-Diez, C. Gonzalez-Guerrero, C. Ocana-Salceda et al., "Calcineurin inhibitors cyclosporine A and tacrolimus induce vascular inflammation and endothelial activation through TLR4 signaling," Scientific Reports, vol. 6, no. 1, article 27915, 2016. 


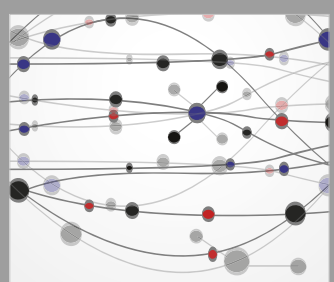

The Scientific World Journal
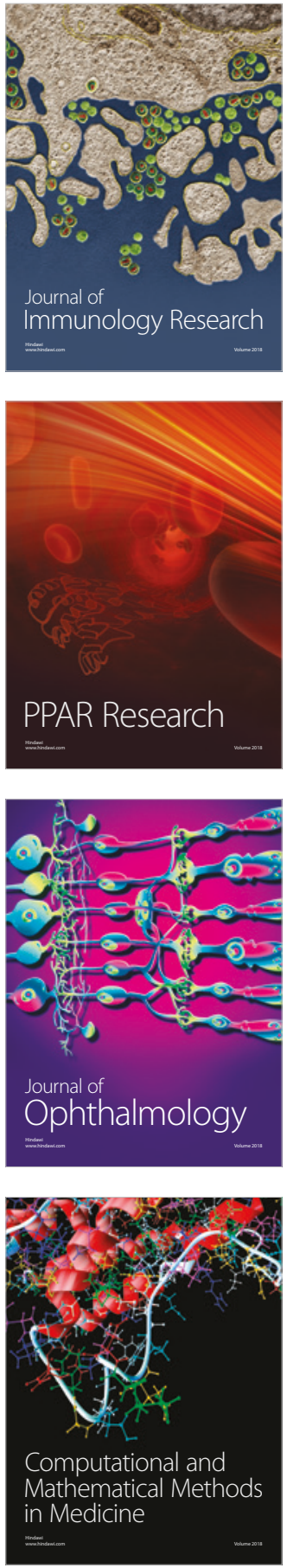

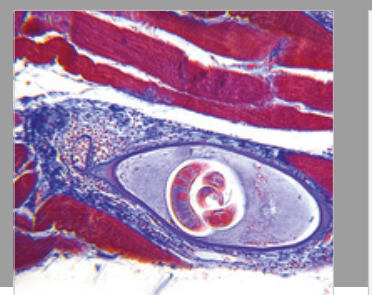

Gastroenterology Research and Practice

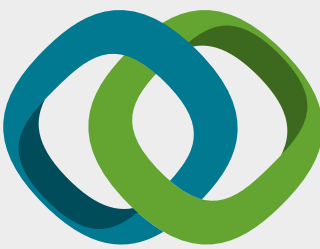

\section{Hindawi}

Submit your manuscripts at

www.hindawi.com
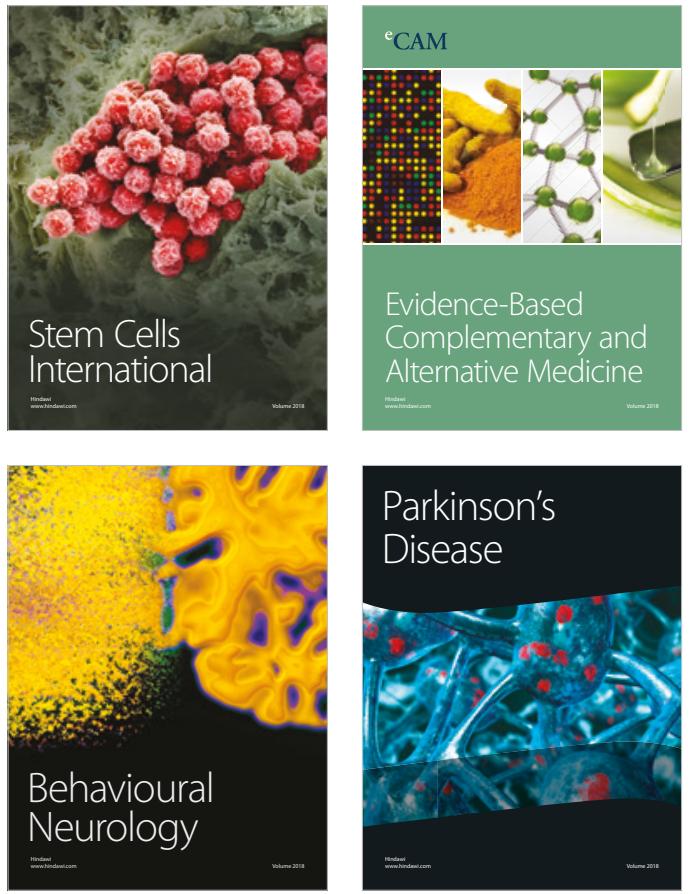

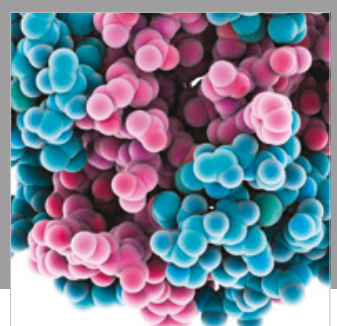

ournal of

Diabetes Research

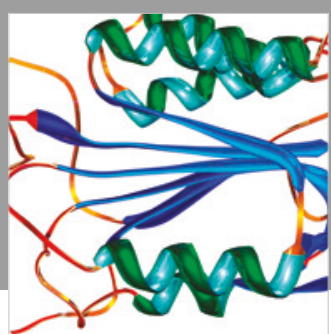

Disease Markers
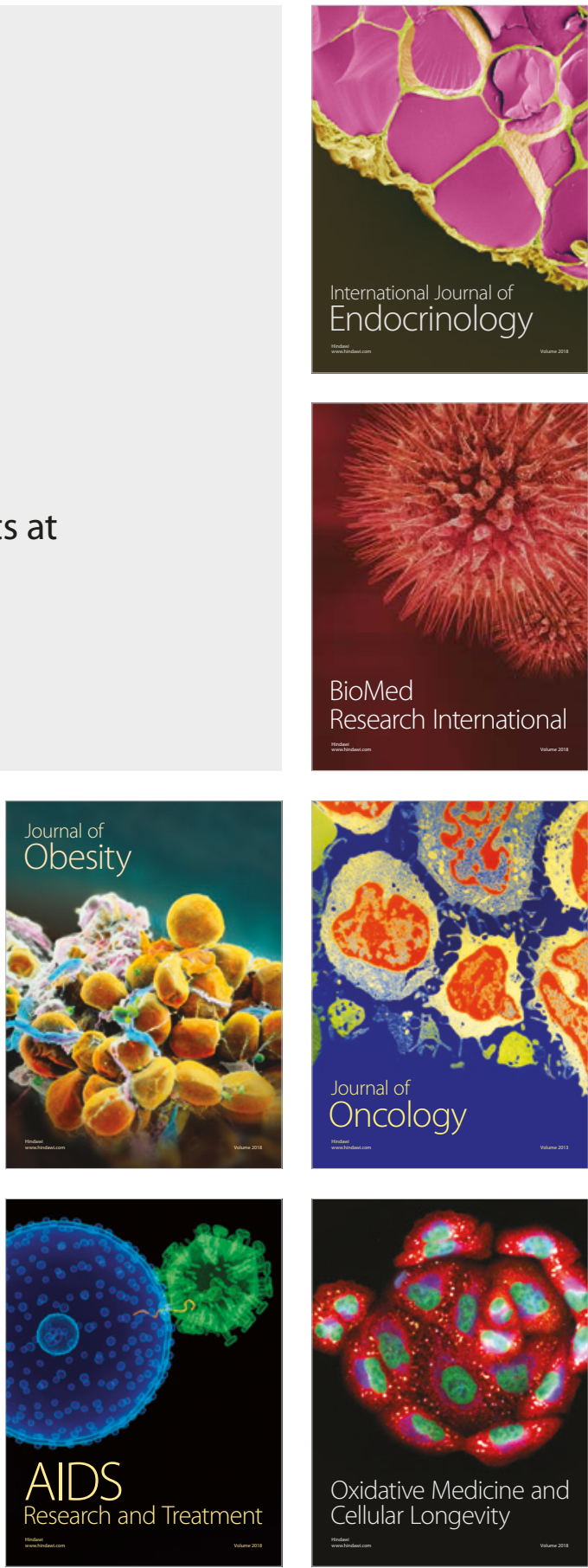